\title{
Pericardial effusion complicated by percutaneous central venous catheterization treated with pericardial window in a very low birth weight infant
}

\author{
Bong Hun Lee, Cheol Hwan So, Seung Hyun Lee, Seung Taek Yu \\ Department of Pediatrics, Wonkwang University School of Medicine, Iksan, Korea
}

Received February 10, 2020

Revised March 9, 2020

Accepted March 9, 2020

Corresponding author

Seung Taek Yu

Department of Pediatrics,

Wonkwang University College of

Medicine, 460 lksan-daero, Iksan

54538, Korea

Tel: $+82-63-859-1510$

Fax: +82-63-853-3670

E-mail: yudoc@wku.ac.kr

ORCID:

https://orcid.org/0000-0001-9744-5548

\begin{abstract}
Percutaneous central venous catheters (PCVCs) provide parenteral nutrition to extremely low birth weight infants. Incorrect PCVC tip location and hyperosmolarity of total parenteral nutrition may lead to pericardial effusion, which has a high mortality rate. When imaging shows a curvature or loop in the catheter tip, pericardial effusion must be considered. Emergency pericardiocentesis and surgical pericardial window as treatments for pericardial effusion show no differences in mortality and complication rates in adults, but no studies have been performed in neonates. Here, we report a very low birth weight infant with pericardial effusion treated by surgical pericardial window.
\end{abstract}

Keywords: Pericardial effusion; Pericardial window techniques; Pericardiocentesis; Very-lowbirth-weight infant

\section{INTRODUCTION}

Percutaneous central venous catheters (PCVCs) are commonly used to provide parenteral nutrition to extremely low birth weight infants in intensive care units. Major complications of PCVCs include occlusion, phlebitis, and sepsis [1]. Pericardial effusion and cardiac tamponade are relatively rare complications, but they can be fatal. Incorrect location of the PCVC tip and hyperosmolarity of total parenteral nutrition (TPN) can contribute to pericardial effusion [2,3]. Treatment options include emergency pericardiocentesis and surgical pericardial window. Several studies have shown no significant difference in adult mortality between the two techniques, although surgical pericardial window has the advantage of less re-accumulation of effusion and retreatent [4]. However, there are no studies evaluating the differences between these techniques in newborns. Here, we report a very low birth weight infant with pericardial effusion caused by an abnormal PCVC tip position and administration of TPN, who was successfully treated by surgical pericardial window technique.

\section{CASE REPORT}

A male neonate, weighing $1,220 \mathrm{~g}$, was born at $286 / 7$ weeks gestational age by Cesarean section due to uncon- 
trolled labor pain. The mother had a previous pregnancy that resulted in a normal delivery. Gestational diabetes was well-controlled by diet, and there was no family history of hereditary diseases. Right after birth, the Apgar scores for the infant were 1 at both 1 and $5 \mathrm{~min}$, and the heart rate was 40 beats/min with severe cyanosis. Positive pressure ventilation was performed with no improvement in symptoms. After tracheal intubation, the Apgar score increased to 5 at $10 \mathrm{~min}$ and 7 at $15 \mathrm{~min}$, and the newborn was transferred to the neonatal intensive care unit (NICU). The birth weight $(1,220 \mathrm{~g})$, height $(38.5 \mathrm{~cm})$, and head circumference $(26.5 \mathrm{~cm})$ were all in the $50-75$ th percentile. On admission to the NICU, body temperature was $36.2^{\circ} \mathrm{C}$, blood pressure $45 / 25 \mathrm{mmHg}$, pulse rate $174 / \mathrm{min}$, and respiration rate $25 /$ min. Neonatal reflex was reduced to a minimum. After entering the NICU, laboratory findings showed $\mathrm{Hb} 16.3 \mathrm{~g} /$ dL, WBC 9,590/ $\mathrm{LL}$ (neutrophils 28.3\%, lymphocytes 56.6\%), platelets 212,000/ $\mathrm{L}$, and serum glucose $46 \mathrm{mg} / \mathrm{dL}$. Arterial blood testing showed $\mathrm{pH} 7.36, \mathrm{pO}_{2} 37 \mathrm{mmHg}, \mathrm{pCO}_{2} 40$ $\mathrm{mmHg}, \mathrm{HCO}_{3}^{-} 19.8 \mathrm{mM} / \mathrm{L}$, with base-excess -6 . There were no specific findings in TORCH (toxoplasmosis, rubella cytomegalovirus, herpes simplex, and HIV) culture tests or on chest X-ray images.

Since birth, the patient had feeding intolerance. On the second hospitalization day, fluid therapy and TPN (glucose: $10 \%$, amino acid: $1.5 \mathrm{~g} / \mathrm{kg}$, and lipid: $1.0 \mathrm{~g} / \mathrm{kg}$ ) were initiated. A PCVC (Premicath ${ }^{\circledR}$, Vygon, Ecouen, France) was placed through the cephalic vein at the radial side of the left antecubital fossa. The procedure was performed with maximal barrier precaution including a cap, mask, sterile gown, sterile gloves, and a large sterile drape [1]. On post-surgical chest radiography, we confirmed that the tip of the catheter was located in the inferior vena cava (IVC). Follow-up chest radiography, on the third hospitalization day, showed that
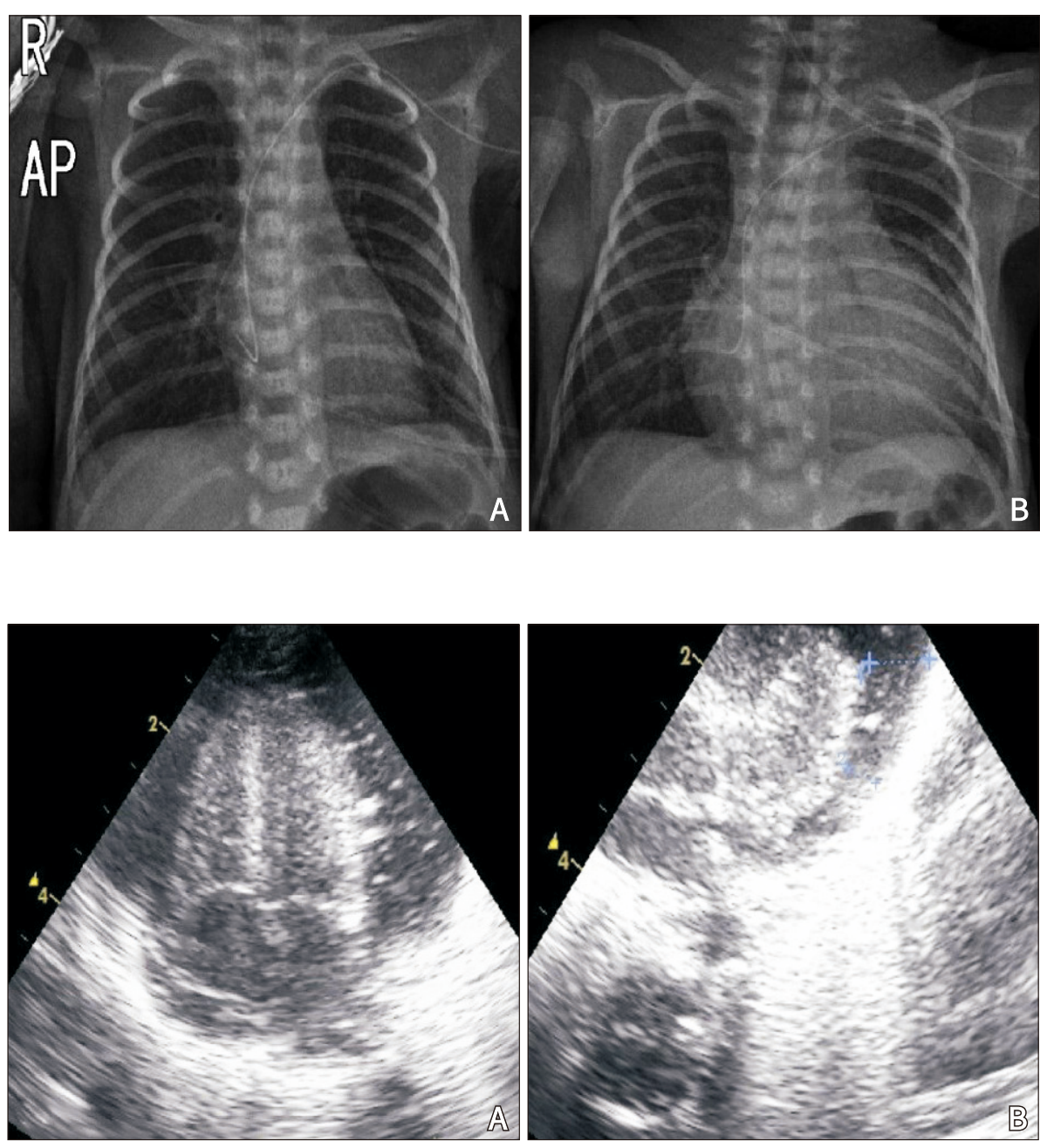

Fig. 2. Echocardiography showing. (A) The apical four-chamber view with pericardial effusion and an ejection fraction of $64.81 \%$, (B) The apical four-chamber ultrasound view showing moderate pericardial effusion with fluid collection between the epicardium and pericardium $(3.10 \mathrm{~mm}$ and $6.05 \mathrm{~mm}$ spaces, respectively). 
the catheter tip was $2 \mathrm{~cm}$ below the diaphragm. We withdrew the catheter by $2 \mathrm{~cm}$ and re-fixed it, confirming that the end of the catheter was located in the right atrium. In a series of chest radiographs, the end of the catheter formed a hook-like figure on the fourth day and a remarkable cardiomegaly was observed on day 6 (Fig. 1). The patient had respiratory support by a ventilator with synchronized intermittent mandatory mode, and his vital signs showed a body temperature of $36.7^{\circ} \mathrm{C}$, respiratory rate $51 / \mathrm{min}$, pulse rate $134 / \mathrm{min}$, and blood pressure 44/26 mmHg. Electrocardiography showed no specific finding, but echocardiography revealed pericardial effusion and a normal ejection fraction of $64.8 \%$ (Fig. 2). We removed the PCVC immediately and recatheterized through the greater saphenous vein. The patient was maintained on conservative treatment with limited fluids and administration of diuretics and inotropics. After four days of treatment, there was no improvement in signs, symptoms, or echocardiographic findings. To reduce the risk of re-accumulation of effusion and the repetition of these procedures, general anesthesia was administered and pericardial window performed through the fifth intercostal space of the anterior axillary line under thoracoscopic assistance on day 10. About $8 \mathrm{~mL}$ of light yellow-colored pericardial effusion was aspirated (Fig. 3). Laboratory results showed a high level of triglycerides ( $438 \mathrm{mg} / \mathrm{dL}$ ), with no specific findings in bacterial cultures and viral polymerase chain reaction (PCR) including cosakievirus, herpes virus. There were no specific findings in the pericardial tissue bi-

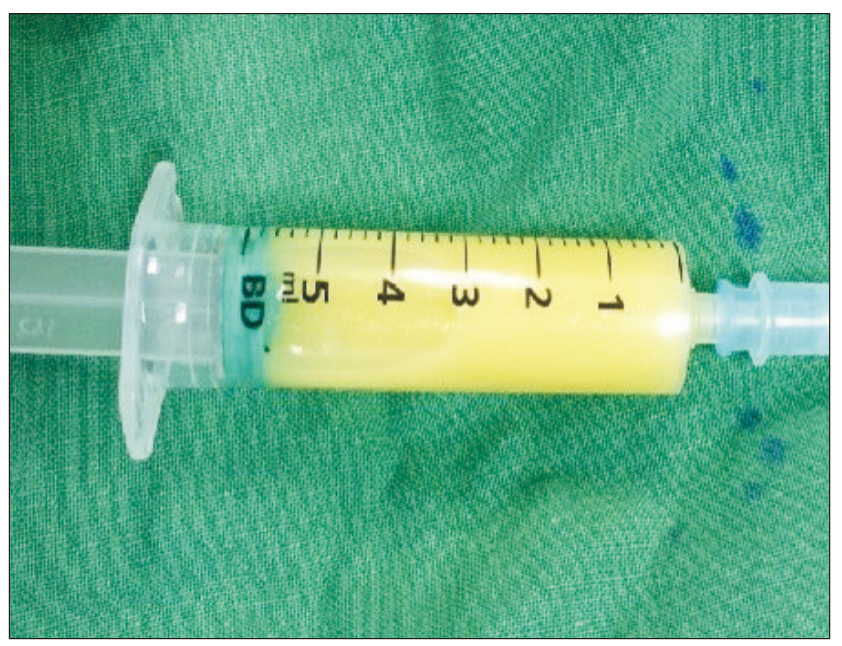

Fig. 3. Pericardial effusion removal from the pericardial sac via the pericardial window. opsy equally. A series of postoperative chest radiographies and echocardiographies confirmed that the pericardial effusion disappeared. The patient had a continuously fixed bowel loop in radiographic images, with feeding intolerance lasting up to hospital day 55, so he was transferred to another hospital for exploratory laparotomy.

The patient's parent provided informed consent for the publication of data and images.

\section{DISCUSSION}

PCVC is widely used in NICUs to maintain intravenous fluid for an extended time in very low birth weight infants. For extremely low birth weight infants, cord veins are available in the first week of birth only, but their use is associated with a higher complication rate when compared to PCVC [5]. Common side effects of PCVC include thrombosis, infection, catheter occlusion, phlebitis, and chronic venous insufficiency. The rates of cardiac tamponade and pericardial effusion are between $0.1 \%$ and $2 \%$, but the associated mortality rates are very high at $34 \%$ and $60 \%$, respectively $[1,2,0]$. Several studies have been conducted on the accurate location of the PCVC tip, which in general is not in the heart but contained within the superior vena cava (SVC) or IVC. Goldwasser et al. [7] reported that the complication rate is twice as high when the PCVC tip is located peripherally rather than centrally with respect to the heart. In addition, higher complications arise from access via the upper than the lower extremities. However, the access via the upper extremity is more common since more veins are available [8]. Pericardial effusion has been reported in other cases where the PCVC tip had formed a curvature or loop [2,9]. In one case, access was made from the upper extremities and a pericardial effusion occurred after the PCVC tip showed curvature [9]. According to Darling et al. [2], curvature in their case occurred when the PCVC tip was pressed against the atrium wall.

The factors affecting pericardial effusion include hyperosmolarity of TPN, thinness of the atrial wall, and the repetitive pulse that resists the catheter tip, resulting in inflammation and tissue necrosis, and eventually causing perforation and cardiac tamponade. Along with location of the PCVC tip, other proposed risk factors for pericardial effusion and cardiac tamponade include the length of catheter with- 
drawal, age of the neonate, and catheter-related infection. However, study results are conflicting [3].

In a study by Nowlen et al. [6], it took about 3 days to detect and diagnose pericardial effusion in 61 patients after a PCVC procedure. It is possible that different mechanisms of pericardial effusion have variable effects, and TPN hyperosmolality may cause greater osmotic injury to the endocardium than direct mechanical trauma. According to several Korean reports, the time to pericardial effusion occurrence was between 16 and 48 hours after catheterization [9,10]. In this case, the diagnosis was made about 60 hours after catheterization, which was relatively late, but since the vital signs were stable and the exudation occurred slowly, a conservative treatment was initially attempted. There was no further clinical deterioration after the initial conservative treatment, except for only a slight change in the exudation, so elective pericardial window treatment was possible. Among 60 infants diagnosed with pericardial effusion/cardiac tamponade in the study by Beardsall et al. [11], 47 patients (78\%) were treated with pericardiocentesis, whereas nine (15\%) improved under conservative treatment. In Korea, all three reported cases were treated with emergency pericardiocentesis. Although the rates of recurrence and repeated procedures are low with pericardial window technique, the burden of general anesthesia should be considered [4]. In this case, we successfully prevented a recurrence by allowing continuous drainage through the inserted chest tube on the elective pericardial window.

Although PCVC is commonly used in NICUs, it is not easy to position the catheter in the correct location due to factors such as venospasm, venous tortuosity, and venous valves [12]. This case highlights the importance of the location of the catheter tip when performing PCVC procedures. When a curvature or loop in the PCVC is visible on imaging, the possibility of pericardial effusion must be considered. Treating pericardial effusion with a surgical pericardial window may reduce recurrence and the need for repeat procedures [4]. As shown in this case, a surgical pericardial window can be a good treatment option for pericardial effusion in premature infants whose vital signs do not fluctuate rapidly.

\section{ACKNOWLEDGMENTS}

We thank Young Min Jo, Sung Bae Jeon for assistance with manuscript preparation.

\section{CONFLICT OF INTEREST}

No potential conflict of interest relevant to this article was reported.

\section{REFERENCES}

1. Ohki Y, Maruyama K, Harigaya A, Kohno M, Arakawa H. Complications of peripherally inserted central venous catheter in Japanese neonatal intensive care units. Pediatr Int 2013;55:185-9.

2. Darling JC, Newell SJ, Mohamdee O, Uzun O, Cullinane CJ, Dear PR. Central venous catheter tip in the right atrium: a risk factor for neonatal cardiac tamponade. J Perinatol 2001;21:461-4.

3. Jumani K, Advani S, Reich NG, Gosey L, Milstone AM. Risk factors for peripherally inserted central venous catheter complications in children. JAMA Pediatr 2013;167:429-35.

4. Horr SE, Mentias A, Houghtaling PL, Toth AJ, Blackstone $\mathrm{EH}$, Johnston DR, et al. Comparison of outcomes of pericardiocentesis versus surgical pericardial window in patients requiring crainage of pericardial effusions. Am J Cardiol 2017;120:883-90.

5. Dongara AR, Patel DV, Nimbalkar SM, Potana N, Nimbalkar AS. Umbilical venous catheter versus peripherally inserted central catheter in neonates: a randomized controlled trial. J Trop Pediatr 2017;63:374-9.

6. Nowlen TT, Rosenthal GL, Johnson GL, Tom DJ, Vargo TA. Pericardial effusion and tamponade in infants with central catheters. Pediatrics 2002;110:137-42.

7. Goldwasser B, Baia C, Kim M, Taragin BH, Angert RM. Non-central peripherally inserted central catheters in neonatal intensive care: complication rates and longevity of catheters relative to tip position. Pediatr Radiol 2017;47:1676-81.

8. Wrightson DD. Peripherally inserted central catheter complications in neonates with upper versus lower extremity insertion sites. Adv Neonatal Care 2013;13:198-204.

9. Kwak KJ, Park JH, Choi HJ, Kim CS, Lee SL. Early-onset pericardial effusion after peripherally inserted central venous catheterization in a preterm infant. Korean J Perinatol 2015;26:355-9.

10. Yang SR, Shin HB, Lee NM, Yi DY, Kim H, Yun SW, et al. Central venous catheter-related cardiac tamponade in premature infants: a report of two cases and a literature review. Korean J Perinatol 2015;26:250-4.

11. Beardsall K, White DK, Pinto EM, Kelsall AW. Pericardial effusion and cardiac tamponade as complications of neonatal 
long lines: are they really a problem? Arch Dis Child Fetal Neonatal Ed 2003;88:F292-5.

12. Racadio JM, Doellman DA, Johnson ND, Bean JA, Jacobs
BR. Pediatric peripherally inserted central catheters: complication rates related to catheter tip location. Pediatrics 2001;107:E28. 